\title{
1 A new oomycete metabarcoding method using the rps10 gene
}

2

3

4

5

6

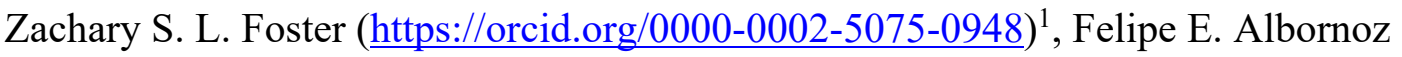

(https://orcid.org/0000-0001-9526-0945) $)^{1 *}$, Valerie J. Fieland (https://orcid.org/0000-00028702-0412) ${ }^{1}$, Meredith M. Larsen², F. Andrew Jones (https://orcid.org/0000-0003-21349888$)^{1,3}$, Brett M. Tyler (https://orcid.org/0000-0003-1549-2987) ${ }^{1,4}$, Hai D. T. Nguyen (https://orcid.org/0000-0002-4764-3009) ${ }^{5}$, Treena I. Burgess (https://orcid.org/0000-00027962-219X) ${ }^{6}$, Carolyn Riddell (https://orcid.org/0000-0003-0089-9135) ${ }^{7}$, Hermann Voglmayr ${ }^{8}$, Frank N. Martin (https://orcid.org/0000-0002-8050-1248) ${ }^{9}$, and Niklaus J.

Grünwald (https://orcid.org/0000-0003-1656-7602) $)^{2}$ \section{(11}

${ }^{1}$ Department of Botany and Plant Pathology, Oregon State University, Corvallis, OR 97331,

\section{USA}

${ }^{2}$ Horticultural Crops Research Unit, Agricultural Research Service, USDA, Corvallis, OR 97330, USA

${ }^{3}$ Smithsonian Tropical Research Institute, Balboa, Panama

${ }^{4}$ Center for Genome Research and Biocomputing (now named Center for Quantitative Life Sciences), Oregon State University

${ }^{5}$ Agriculture and Agri-Food Canada, Ottawa, ON, K1A 0C6, Canada

${ }^{6}$ Phytophthora Science and Management, Harry Butler Institute, Murdoch University,

21 Murdoch, Australia

${ }^{7}$ Forest Research, Roslin, Edinburgh, EH25 9SY, UK 
${ }^{8}$ Institute of Forest Entomology, Forest Pathology and Forest Protection, Department of Forest and Soil Sciences, BOKU-University of Natural Resources and Life Sciences, Vienna, Austria

${ }^{9}$ Crop Improvement and Protection Research, Agricultural Research Service, USDA, Salinas, CA 93905, USA

* Current address: CSIRO Land and Water, Wembley, WA, Australia

Corresponding authors:

Frank Martin, frank.martin@usda.gov

Niklaus Grünwald, nik.grunwald@usda.gov

\section{Interpretive summary}

Oomycetes are a group of eukaryotes related to brown algae and diatoms, many of which cause diseases in plants and animals. Improved methods are needed to rapidly characterize the diversity of oomycete species found in environmental samples. We have identified the mitochondrial 40S ribosomal protein S10 gene (rps10) as being useful for oomycete community sequencing. We evaluated its utility relative to a popular barcode, the internal transcribed spacer 1 (ITS1), by sequencing environmental samples and a community we synthesized in the laboratory. The amplified $r p s 10$ region is predicted to have a higher taxonomic resolution than ITS1, allowing for greater discrimination of closely related species.

4 We present a new website with a searchable $r p s 10$ reference database for species 
barcode and methods described herein provide an effective tool for characterizing oomycetes using environmental DNA sequencing.

\section{Abstract}

Oomycetes are a group of eukaryotes related to brown algae and diatoms, many of which cause diseases in plants and animals. Improved methods are needed for rapid and accurate characterization of oomycete communities using DNA metabarcoding. We have identified the mitochondrial 40S ribosomal protein S10 gene (rps10) as a locus useful for oomycete metabarcoding and provide primers predicted to amplify all oomycetes based on available reference sequences from a wide range of taxa. We evaluated its utility relative to a popular barcode, the internal transcribed spacer 1 (ITS1), by sequencing environmental samples and a mock community using Illumina MiSeq. Amplified sequence variants (ASVs) and operational taxonomic units (OTUs) were identified per community. Both the sequence and predicted taxonomy of ASVs and OTUs were compared to the known composition of the mock community. Both rps 10 and ITS yielded ASVs with sequences matching 21 of the 24 species in the mock community and matching all 24 when allowing for a $1 \mathrm{bp}$ difference. Taxonomic classifications of ASVs included 23 members of the mock community for rps 10 and 17 for ITS1. Sequencing results for the environmental samples suggest the proposed rps 10 locus results in substantially less amplification of non-target organisms than the ITS1 method. The amplified rps10 region also has higher taxonomic resolution than ITS1, allowing for greater discrimination of closely related species. We present a new website with a searchable rps 10 reference database for species identification and all protocols needed for oomycete 
metabarcoding. The rps 10 barcode and methods described herein provide an effective tool for metabarcoding oomycetes using short-read sequencing.

Key words: amplicon metagenomics, ITS1, metabarcoding, DNA, detection, ecology, water mold, Illumina, MiSeq

\section{INTRODUCTION}

\section{Oomycetes are microscopic eukaryotes related to brown algae and diatoms that often cause} diseases in plants and animals (Baldauf, Roger, Wenk-Siefert, \& Doolittle, 2000; Yoon, Hackett, Pinto, \& Bhattacharya, 2002). They include highly destructive pathogens with major impacts on agriculture (Fry, 2008), aquaculture (Phillips, Anderson, Robertson, Secombes, \& van West, 2008), and natural ecosystems (Cahill, Rookes, Wilson, Gibson, \& McDougall, 2008; Grünwald, LeBoldus, \& Hamelin, 2019). Oomycetes that cause agricultural diseases include: Phytophthora infestans, the cause of potato late blight and the Irish Potato Famine (Fry, 2008); Aphanomyces euteiches, the pathogen responsible for damping-off and root rot of legumes (Gaulin, Jacquet, Bottin, \& Dumas, 2007); Pythium species that cause damping-off and root rot on a large variety of agricultural and horticultural crops (F. N. Martin \& Loper, 1999); and host-specific obligate pathogens known as the white rusts and downy mildews (Spring et al., 2018).

In addition to their impacts on agriculture, invasive oomycete pathogens have destructive effects on forests, managed landscapes, and aquatic ecosystems (Hansen, Reeser, \& Sutton, 2012). Forests in North America and Europe have suffered significant tree mortality due to 
sudden oak death and larch death, respectively, caused by Phytophthora ramorum (Brasier \& Webber, 2010; Grünwald, Goss, \& Press, 2008). Eucalypt forests in Australia are experiencing massive dieback caused by invasive Phytophthora cinnamomi (Burgess et al., 2017). Tree seedlings in natural ecosystems regularly suffer damping off, which is frequently associated with Pythium species (Augspurger \& Wilkinson, 2007). Some oomycetes are also significant fish and crustacean pathogens, such as Saprolegnia parasitica and Aphanomyces invadans, and are of great concern in aquaculture (van West, 2006). Because of the ecological and economic impacts of oomycetes, improving methods to characterize the distribution of these pathogens quickly, accurately, and economically would help control and understand this important, but relatively understudied, group of organisms.

Although many oomycete pathogens are well known for the extensive damage they cause to natural ecosystems and agriculture (Wills, 1993), the global diversity and distribution of oomycetes as a whole is less well characterized compared to other microorganisms, such as fungi and bacteria. This is due, in part, to the challenge of collecting samples during periods when oomycetes are active, isolating them from diverse host and substrate materials (e.g., water, soil, plant, and animal tissues), culturing them on an assortment of specialized media (or in vivo for the numerous obligate pathogens), and identifying species using morphological characteristics. Methods relying on DNA sequencing are increasingly used to complement or replace these traditional techniques. Culture-independent DNA-based methods like metabarcoding have the potential to overcome many of the challenges associated with characterizing oomycete communities (Salcedo et al., 2021; Tedersoo, Drenkhan, Anslan, Morales-Rodriguez, \& Cleary, 2019), as has been demonstrated with fungi (White et al., 1990; Nilsson et al., 2019; Schoch et al., 2012) and bacteria (Bukin et al., 2019; Tringe \& 
117 Hugenholtz, 2008). In order for metabarcoding of oomycetes to be effective, a region of

118 DNA ideally should: (1) contain enough variability to differentiate isolates to species level,

119 (2) be small enough for short-read, high-throughput sequencing, and (3) be flanked by regions

120 where primers can be designed that are conserved in oomycetes, but diverged in other

121 organisms (Cristescu, 2014). In addition, a curated database of high-quality reference

122 sequences of the barcode region must be publicly available so environmental sequences

123 produced by metabarcoding can be assigned taxonomic classifications. In practice, it is

124 difficult to find a barcode with all these properties. Many widely used barcodes/primers fail to

125 differentiate closely related species, often amplify non-target organisms, or fail to amplify

126 target organisms.

127

128 Reads generated by metabarcoding are generally clustered into operational taxonomic units

129 (OTUs) or amplified sequence variants (ASVs). Both methods serve to mitigate sequencing

130 error by grouping similar sequences. However, OTUs are created by clustering at a specific

131 sequence similarity threshold, usually meant to simulate species-level differences, whereas

132 ASVs are created using a statistical model of mutation and read abundance to correct for

133 sequencing errors (Callahan et al., 2016). OTU clustering is the older and more established of

134 the two methods. The threshold used for OTU clustering is specific to the locus and group of

135 organisms analyzed. This threshold is somewhat arbitrary, considering that different OTU

136 clustering techniques apply this threshold differently (Chen, Zhang, Cheng, Zhang, \& Zhao,

137 2013), but is designed to simulate species-level differences. ASV inference requires no pre-

138 determined clustering threshold, but rather similar sequences are grouped to the extent needed

139 to correct for sequencing errors based on a model of mutation frequencies inferred from the

140 data. Ideally, ASVs represent real biological sequences and can be compared between studies, 
141 whereas OTUs are emergent properties of a specific dataset and cannot be compared between

142 studies (Callahan, McMurdie, \& Holmes, 2017). ASV inference is a relatively new but

143 currently preferred method due to it robust statistical foundation and ability to produce results

144 that are comparable across studies.

146 There have been several published DNA barcodes for oomycetes (Choi et al., 2015; Robideau

147 et al., 2011; Yuan, Feng, Zhang, \& Zhang, 2017). However, the most popular, the internal

148 transcribed spacer 1 of the ribosomal DNA (ITS1), has insufficient taxonomic resolution to

149 identify many oomycetes to the species level (Redekar, Eberhart, \& Parke, 2019), which can

150 lead to ambiguous or incorrect taxonomic classifications (Riddell et al., 2019). Furthermore,

151 currently available ITS1 primers amplify distantly related non-target organisms such as plants

152 and fungi (Coince et al., 2013) or only amplify some oomycete genera (Legeay et al., 2019).

153 A widely used method uses a semi-nested PCR with the primers ITS6 and ITS4 in the first

154 reaction followed by ITS6 and ITS7 in the subsequent reaction (Cooke, Drenth, Duncan,

155 Wagels, \& Brasier, 2000). In practice, as little as 5.3\% of the OTUs (57\% of reads) recovered

156 using this method were assigned to oomycetes (Coince et al., 2013). Sapkota and Nicolaisen

157 (2015) proposed increasing the annealing temperature to increase specificity to oomycetes and

158 reported $60 \%$ of OTUs (95\% of reads) assigned to oomycetes. Riit et al. (2016) developed

159 primers targeting the ITS1 and ITS2 regions without the need for a semi-nested approach;

160 those primers enabled assignment of $22 \%$ and $29 \%$ of OTUs to oomycetes $(25 \%$ and $30 \%$ of

161 reads, respectively). Other primers for ITS1 and other loci, including cytochrome c oxidase

162 subunit I ( coxl) and cytochrome c oxidase subunit II ( $\operatorname{cox} 2)$, have been used, but have either

163 not been extensively tested or only target the genus Phytophthora (Esmaeili Taheri,

164 Chatterton, Gossen, \& McLaren, 2017; Fiore-Donno \& Bonkowski, 2021; Landa et al., 2021; 
165 Riddell et al., 2019; Sapp et al., 2016). In summary, all the ITS1-based methods targeting oomycetes we are aware of have shortcomings regarding non-target amplification and/or taxonomic resolution.

The mitochondrial rps 10 gene, encoding the 40S ribosomal protein has been helpful in delineating species and estimating phylogenetic relationships in Phytophthora (F. N. Martin, Blair, \& Coffey, 2014). Preliminary analysis using mitochondrial genomes from a broad range of taxa identified an arrangement of tRNA genes flanking the rps 10 gene unique to oomycetes (tRNA-Phe, rps10, tRNA-Arg, tRNA-Gln, tRNA-Ile, tRNA-Val; F. Martin,

174 unpublished). Amplification primers designed from conserved regions of $t R N A-P h e$ and $175 t R N A$-Ile amplified templates suitable for sequencing from many different oomycete species 176 (Martin et al., 2014; F. N. Martin, unpublished). While this locus was useful for phylogenetic analysis and as a barcode for species identification, a mean length of approximately $600 \mathrm{bp}$ makes it too long for metabarcoding using Illumina sequencers. Yuan et al. (2017) also noted

179 the sequence divergence of the rps10 locus (among others) for 14 oomycete taxa and

180 suggested the locus as a candidate barcode for oomycetes.

182 Here, we propose the rps 10 locus as an oomycete barcode and provide primers suitable for

183 short-read sequencing on platforms like the Illumina MiSeq. The usefulness of $r p s 10$ primers

184 was compared to the semi-nested method to amplify ITS1 proposed by Sapkota and

185 Nicolaisen (2015), which has reported some of the best results using ITS1 for oomycete

186 metabarcoding. We compared each method's taxonomic specificity and resolution by

187 simulating PCR amplification with reference sequences and conducting metabarcoding of 188 environmental samples and a mock community composed of a mixture of known species 
189

190

191

192

193

194

195

196

197

198

199

200

201

202

203

composition. We clustered reads into both ASVs and OTUs to determine which approach works best with the proposed method. We developed a companion website to host the rps10 reference database and describe all protocols needed for researchers to immediately apply this validated method to oomycete metabarcoding.

\section{MATERIALS AND METHODS}

2.1 Primer design

Metabarcoding primers suitable for use with the Illumina MiSeq (San Diego, CA) were designed by searching sequence alignments of the region around the rps 10 locus. The sequences consisted of amplicons generated using primers in the flanking $t R N A-P h e$ and tRNA-Ile loci (F. N. Martin et al., 2014) or extracted from assembled mitochondrial genomes (F. N. Martin, unpublished) and represented 16 genera and 92 species of oomycetes.

Sequences were aligned with Clustal Omega (Sievers et al., 2011) and inspected in Geneious 8.1.9 (Biomatters, Auckland, New Zealand). Forward and reverse primers were designed for the highly conserved regions in the $t R N A$-Phe and $t R N A$-Arg genes flanking the $r p s 10$ gene (Figure 1). Pairs of potential primers for metabarcoding were chosen that would: (1) result in an amplicon length of less than $500 \mathrm{bp}$ and would therefore be appropriate for short-read sequencing using platforms like the Illumina MiSeq, (2) be conserved in all known oomycetes, and (3) not match the sequences of other organisms, particularly fungi and plants (Cristescu, 2014). Potential primers were evaluated with OligoAnalyzer (Owczarzy et al., 2008) to assess their melting temperatures, CG content, and potential for forming problematic 
secondary structures, such as dimers and hairpins. Table 1 provides the primers developed for rps 10 metabarcoding and the sequences found in the reference database.

\subsection{Simulated PCR}

217 To test the newly developed primers for taxonomic specificity and coverage, we used the rps10 locus extracted in silico from the mitochondrial genomes of 121 oomycetes, 38 nonoomycete stramenopiles, 19 fungi from different families, and four Rickettsia species.

Rickettsia species were included because segments of their genomes resemble mitochondrial genomes (Andersson et al., 1998). We evaluated the sensitivity and specificity of the primers using Geneious 8.1.9 with the following parameters: no mismatches allowed, SantaLucia (1998) formula and salt correction, $50 \mathrm{nM}$ concentration of oligos, and $0.6 \mathrm{mM}$ dNTPs. The results were analyzed and visualized in a taxonomic context using the R packages taxa

225 (Foster, Chamberlain, \& Grünwald, 2018) and metacoder (Foster, Sharpton, \& Grünwald, 226 2017)

230 To test for amplification of oomycetes and taxonomic resolution using the metabarcoding

231 primers, a DNA mixture representing a mock community of 24 oomycete species was

232 prepared (Table S1). The mock community was composed of a mixture of known oomycete 233 species from different laboratories. The DNA was extracted from cultured strains or host

234 tissue (for obligate pathogens) depending on species (Table S1). DNA from each of the 24 235 species was pooled, resulting in a per-species final concentration of $\sim 2 \mathrm{ng} / \mu \mathrm{L}$, with the 
exception of a lower concentration for Saprolegnia diclina $(0.5 \mathrm{ng} / \mu \mathrm{L})$ and Phytophthora pluvialis $(1.0 \mathrm{ng} / \mu \mathrm{L})$. The concentration of DNA measured represented all the DNA in the sample; thus, DNA extracts from infected plant tissue (for obligate pathogens) included an unknown proportion of plant DNA and an unknown concentration of oomycete DNA.

To test for non-target amplification, we extracted DNA from diverse environmental samples including soil, water, and plant tissue. Samples consisted of soils from Panama (Schappe et al., 2017) and soil, canopy drip water, and tree needles collected near old-growth Pseudotsuga menziesii in the Wind River Forest Dynamics Plot in Washington State, USA. DNA from soil was extracted following a previously reported protocol (Schappe et al., 2017). DNA from needles was extracted using the DNeasy Plant Mini Kit (Qiagen, Valencia, CA, USA), following the manufacturer's instructions. Water samples were collected by filtering up to $1 \mathrm{~L}$

248 of water dripping through tree canopies with $0.45 \mu \mathrm{m}$ filters and extracting DNA from the 249 filters using the DNeasy Plant Mini Kit.

2.4 DNA amplification and high-throughput sequencing

DNA from the mock community and environmental samples was amplified with both the rps 10 method and the ITS1 method (Sapkota \& Nicolaisen, 2015) to generate amplicons for high-throughput sequencing. Negative PCR water controls were included in both assays. The rps 10 assay is a multiplex PCR reaction comprising two rps 10 forward primers that differ

257 slightly in sequence but anneal to the same position in the $t R N A-P h e$ gene (rps10_F1 and rps10_F2) and seven rps10 reverse primers that differ slightly in sequence but anneal to the 
amplifications of the rps10 locus were carried out using the QIAGEN Type-it Mutation

Detect PCR Kit (QIAGEN, 206343, Valencia, CA). Multiplex PCR reactions were performed

T) representing two primers and was therefore added at a final concentration of $0.4 \mu \mathrm{M}$.

Amplifications were carried out in a Veriti thermal cycler (Life Technologies, Grand Island,

$\mathrm{NY}$ ) with an initial denaturation at $95^{\circ} \mathrm{C}$ for $5 \mathrm{~min}$, followed by 35 cycles of $95^{\circ} \mathrm{C}$ for $30 \mathrm{~s}$,

$58^{\circ} \mathrm{C}$ for $3 \mathrm{~min}$, and $72^{\circ} \mathrm{C}$ for $30 \mathrm{~s}$, and a final extension at $60^{\circ} \mathrm{C}$ for $30 \mathrm{~min}$.

ITS1 was amplified following a semi-nested protocol with minor modifications from the previously published method using the ITS6/ITS4 and ITS6/ITS7 primer sets (Sapkota \& previous efforts to use various proofreading Taq polymerases resulted in unacceptably strong amplification of plant DNA using these primers (unpublished data), possibly due to the 3' to 5' exonuclease activity of proofreading Taq polymerase removing bases at the 3 ' end of the

277 ITS7 primer that distinguishes oomycetes from plants (Sapkota \& Nicolaisen, 2015). DNA

278 (30 ng, except for P. pluvialis at $15 \mathrm{ng}$ and $S$. diclina at $7.5 \mathrm{ng}$ ) was added to each reaction for 279 a total volume of $15 \mu \mathrm{L}$. The second PCR reaction was identical to the first with the following 280 exceptions: the template was $1.0 \mu \mathrm{L}$ of the initial PCR reaction, primers were ITS6 and ITS7, 281 and the total reaction volume was $25 \mu \mathrm{L}$. Both ITS1 PCR amplifications were conducted in a 282 Bio-Rad T100 thermocycler (Bio-Rad, Hercules, CA, USA) under the following thermal 
and a final extension of $2 \mathrm{~min}$ at $72^{\circ} \mathrm{C}$. The protocol used differs slightly from that of Sapkota and Nicolaisen (2015); the first PCR had 25 cycles instead of 15 and the annealing temperatures of the PCR were raised from $55{ }^{\circ} \mathrm{C}$ and $59{ }^{\circ} \mathrm{C}$ to $60{ }^{\circ} \mathrm{C}$ for both. These changes were based on optimizing the PCR conditions to minimize non-target amplification in a previous experiment (Foster, Weiland, Scagel, \& Grünwald, 2020). Amplicons from both the rps 10 and second ITS1 PCR amplicons were then cleaned, ligated to Illumina Nextera XT indices and adapters, purified, and pooled following Illumina manufacturer's protocols University.

2.5 Rps10 database and associated website

A curated reference database was developed for assigning taxonomic classifications to sequences generated from the rps 10 primers. The database is composed of sequences manually curated from online databases, contributed by other research groups, identified from whole mitochondrial genomes, and produced from known isolates in this study. Species attempted to find primers that would produce an amplicon that included both metabarcoding

304 primer binding sites, which would be useful for detecting primer mismatches when new organisms are sequenced in the future, but found it was only possible to include the binding

306 site of the reverse primer. We selected previously reported amplification primers (F. N.

307 Martin et al., 2014) and modified them by addition of degenerate bases. Amplification of the 
rps 10 database amplicon was conducted in $25.0 \mu \mathrm{L}$ reactions with $0.025 \mathrm{U} / \mu \mathrm{L}$ GenScript Taq sequences to the reference database.

318 An ASV abundance matrix with associated taxonomic annotations was created from MiSeq reads using cutadapt (M. Martin, 2011) and the R package dada2 (Callahan et al., 2016).

320 Primer sequences were trimmed from reads using cutadapt. Reads were then filtered out using the filterAndTrim command of dada2 if they were expected to contain 5 or more errors, based on their quality scores. Reads were also truncated at the first instance of a quality score less than 5. Error rates were estimated and used to infer ASVs using the learnErrors and dada commands for each locus. ASV read pairs were merged using the mergePairs function and predicted chimeras were removed using removeBimeraDenovo. Any merged sequences less than $50 \mathrm{bp}$ long were also removed. A taxonomic classification was assigned to each ASV

327 using the RDP Naive Bayesian Classifier algorithm implemented in the assignTaxonomy 328 command (Wang, Garrity, Tiedje, \& Cole, 2007). The algorithm assigns a bootstrap value to 329 each taxonomic rank for each classification, providing a confidence measure for which taxon 330 in the reference database is most similar. For $r p s 10$ sequences, the newly developed $r p s 10$ 331 database described herein was used as the reference database; for ITS1, a combination of 
332 UNITE (Kõljalg et al., 2005), Phytophthora-DB (Park et al., 2008), and sequences from

333 Robideau et al. (2011) were used. Each ASV was also optimally aligned to the best-matching

334 reference sequence to calculate a percent identity using the pairwiseAlignment function from

335 the biostrings R package (Pages, Aboyoun, Gentleman, \& DebRoy, 2017).

337 Reads were also clustered into OTUs using VSEARCH (Rognes, Flouri, Nichols, Quince, \&

338 Mahé, 2016) to create an OTU abundance matrix. To inform the choice of clustering

339 threshold for each locus, ASVs present in the mock community were clustered at a range of

340 thresholds from $90 \%$ to $100 \%$ in $0.1 \%$ increments and the number of resulting OTUs was

341 recorded. Thresholds were chosen that best reproduced the number of species used in the

342 mock community and conformed with previous experience using ITS1 as an oomycete

343 barcode (Foster et al., 2020). Rps10 sequences were clustered at a 96\% threshold and ITS1

344 sequences were clustered at a 99\% clustering threshold. Chimera detection, taxonomic

345 assignment, and calculation of percent identity of OTUs to the assigned reference sequence

346 were done with the same methods used for ASVs described above.

2.7 Mock community

350 The inferred composition of the mock community based on sequencing results was compared

351 with the known composition of the mock community to evaluate the performance of the rps 10

352 and ITS1 methods. For this analysis, only ASVs represented by at least 10 reads were used.

353 ASVs found in the mock community samples were classified as "expected", or "non-target".

354 Three metrics were used to evaluate the ability of each method to infer the composition of the

355 mock community: (1) the number of mock community members detected, (2) the proportion 
of ASVs representing members of the mock community, and (3) the proportion of reads representing members of the mock community. These three metrics were calculated using both the taxonomic classifications and the sequences, resulting in a total of 6 metrics for each locus. Taxonomic classifications were considered correct that contained the species name of a

360 member of the mock community. Sequences were considered correct if they matched the

361 reference sequences of the mock community members exactly, as determined by alignments of each ASV to each reference sequence using the pairwiseAlignment function from the biostrings $\mathrm{R}$ package. The same analysis was done allowing for a single mismatch in an alignment between the ASV and the reference sequence to check for nearly correct sequences.

ASVs and OTUs associated with environmental samples were used to assess non-target amplification. Since our reference databases do not contain sequences for many groups of 370 potential non-target organisms, ASVs and OTUs in this analysis were assigned an alternative 371 taxonomic classification using a BLAST search against the NCBI nucleotide database 372 (Altschul, Gish, Miller, Myers, \& Lipman, 1990). Although NCBI taxonomic annotations can 373 be unreliable (Nilsson et al., 2006), we only considered the kingdom-level part of the 374 taxonomy, which we considered more likely to be correct. The best BLAST hit was chosen 375 for each ASV/OTU based on the E-value and percent identity of the matching region. BLAST 376 hits with an E-value higher than 0.001 were not considered. Using the taxonomy associated 377 with the best BLAST hit, ASVs were grouped into "Oomycetes", "Fungi", and "Other" 
proportions of reads, ASVs, and OTUs in each category for each locus were then compared to evaluate the amount of non-target amplification for each locus.

\subsection{Taxonomic resolution}

The ability of each locus to distinguish different species was evaluated by pairwise alignments of reference database sequences. The portion of the reference database sequences predicted to be amplified by each primer pair was aligned using MAFFT v7.453 (Katoh, 2005) and all pairwise differences in sequence identity were calculated using the ape $\mathrm{R}$ package. Reference sequences with the complete amplicon were used, as determined by the presence of primer binding sites, using a modified version of the matchProbePair function from the Biostrings package that allows for ambiguity codes. Reference sequences were also included if they aligned to at least $90 \%$ of one of the predicted amplicons, in which case the portion aligned to the amplicon was used. Only reference sequences for species present in both databases were used in order to make the comparison as fair as possible. For each locus, the distributions of the percent identity of each species' sequence to the most similar sequence from a different species were compared to assess taxonomic resolution.

\section{The taxonomic resolution was also investigated by comparing the distribution of bootstrap} scores of ASV taxonomic assignments and the bootstrap scores generated by the neighborjoining tree of mock community species. The bootstrap values used were those assigned by the RDP Naive Bayesian Classifier algorithm implemented by the assignTaxonomy command of dada2; these values measure how consistent the taxonomic assignment is for a given reference database when parts of the sequences are subsampled. Bootstrap values were also 
403

404

405

406

407

408

409

410

411

412

413

414

415

416

417

418

420

421

426

generated to assess taxonomic resolution when there were multiple reference sequences for the same species. The distribution of bootstrap values for mock community samples for the genus, species, and reference sequence ranks were compared for ITS1 and rps10. In addition, the bootstrap scores for clades in the neighbor-joining trees of mock community species were compared.

\section{RESULTS}

3.1 Validation of metabarcoding primers for the $\operatorname{rps} 10$ region

The new primers developed for oomycete-specific amplification of the rps 10 locus were validated using simulated PCR of reference sequences (Figure 1). There are two forward primers and seven reverse primers that bind to the same respective regions but differ slightly in sequence (Table 1). Simulated PCR using the forward and reverse primer sets amplified the rps 10 gene with no mismatches on any of the 121 oomycete sequences analyzed (Figure 2 ). In addition, the rps 10 primers were predicted not to amplify any non-target species found in the stramenopiles, fungi, and Rickettsia (Figure 2). The forward primer set has $40 \%$ mean GC content and a predicted mean melting temperature of $59.8^{\circ} \mathrm{C}$, while the reverse primer set has $31 \%$ mean GC content and a predicted mean melting temperature of $58.2^{\circ} \mathrm{C}$. The primers are predicted to amplify a sequence with a median length of $481 \mathrm{bp}$ (including primers), with Albugo laibachii producing the shortest with 448 bp, and Peronospora tabacina producing the longest with 513 bp (Figure S1). The length of amplicons produced by the ITS1 method, inferred using the same procedure, varied from $225 \mathrm{bp}$ to $400 \mathrm{bp}$. 
3.2 The $\operatorname{rps} 10$ reference database and website

To host the rps10 database and laboratory protocols, we created the website

www.oomycetedb.org. The database currently contains 886 sequences representing 346

species and 20 genera of oomycetes (Table 2). The website is a combination of static HTML

BLAST can output. Updates to the database are released on this website with a unique version

441 github.com/grunwaldlab/OomyceteDB.

A mock community composed of DNA from 24 species of known oomycetes was sequenced on the Illumina MiSeq to evaluate the ability of the proposed rps 10 metabarcoding method to

447 infer community composition relative to an ITS1-based method. Both the rps 10 and ITS1

448 methods resulted in more ASVs and OTUs than species included in the mock community.

449 The rps10 method resulted in fewer ASVs than the ITS1 method but more OTUs, suggesting 450 that the ASV-based analysis better controlled for sequencing error than the OTU-based 
analysis (Table 3). The taxonomic classifications of ASVs produced by the rps 10 method included 23 of the 24 mock community species whereas the ITS1 classifications included 17 (Table 3). The species missing in the rps 10 classifications was Phytophthora ipomoeae and the species missing in the ITS1 classifications were Phytophthora citrophthora, Phytophthora himalsilva, Phytophthora ipomoeae, Phytophthora quercina, Pythium dissotocum, Pythium oligandrum, and Pythium undulatum. The rps10 ASVs assigned to species in the mock community accounted for $95 \%$ of all ASVs and $>99.9 \%$ of the reads, whereas the ITS 1 ASVs with correct classifications accounted for $75.6 \%$ of all ASVs and $54.5 \%$ of the reads. Similar metrics were produced by considering whether the correct sequences were found, regardless

460 of taxonomic classification. Both methods resulted in ASV sequences matching 21 of the 24 461 species exactly and matching all 24 when a $1 \mathrm{bp}$ mismatch in the alignment between ASVs and reference sequences was tolerated. The rps10 ASVs with exact sequence matches accounted for $62.5 \%$ of ASVs and $99.3 \%$ of reads, whereas the ITS1 ASVs with correct sequences matches accounted for $73.3 \%$ of ASVs and $99.2 \%$ of reads. In five out of the six metrics used to evaluate how well the mock community composition was inferred, the rps 10 method performed equal to or better than the ITS1 method (Table 3).

A diverse set of environmental DNA extracts were sequenced on the Illumina MiSeq using

471 the proposed rps 10 metabarcoding method to evaluate taxonomic specificity. For the rps 10

472 method, oomycete sequences accounted for $99.5 \%$ of the reads, $84.8 \%$ of the ASVs, and

$47353.5 \%$ of the OTUs (Figure 3). For the ITS1 method, oomycete sequences accounted for $47463.0 \%$ of the reads, $10.7 \%$ of the ASVs, and $5.5 \%$ of the OTUs. Most of the rps 10 sequences 
not assigned to oomycetes could not be assigned to a taxon based on BLAST searches against the NCBI nucleotide database, particularly for the plant-derived samples. Most of the ITS1 sequences not assigned to oomycetes were assigned to fungi and a much smaller proportion were assigned to plants. For both methods non-target ASVs and OTUs were at lower abundance than oomycete sequences, but this trend was more pronounced for rps 10. Manual investigation of the rps10 ASVs with no matches to NCBI revealed that these sequences are much shorter than typical rps 10 sequences, have a much higher GC content, and do not align well to rps10 reference sequences or to each other. Overall, sequencing of amplicons from environmental samples from water, soil, and plant material using the rps 10 barcode resulted in less non-target amplification, in terms of ASV, OTU, and read counts than when using the ITS1 barcode (Figure 3).

\subsection{Taxonomic resolution}

\section{The ability to differentiate closely related species was evaluated by comparing how many}

base pairs differentiated each reference sequence from the most similar sequence for a

different species in the region amplified. In general, the pairwise differences between the most similar sequences from different species were greater for $r p s 10$ than ITS1 (Figure 4A). A total of $14.7 \%$ of the predicted amplicons derived from unique species in the rps 10 reference database shared an identical sequence with a different species, compared with $29.4 \%$ in the ITS1 database (Figure 4B). In addition, $67.6 \%$ of the species in the rps 10 database were distinguished from their most closely related species by five or more base pairs, in contrast to only $16.2 \%$ for ITS 1 . Polymorphic sites in the rps 10 locus are distributed across the entire length of the amplicons and sequences align with few gaps. In contrast, variation in the ITS1 
locus appears in clusters and alignments have frequent large indels in many blocks (Figure 5).

500 Another way to compare the ability to distinguish sequences is the distribution of bootstrap

501 scores for taxonomic assignment and sequence clustering of the mock community sequences.

502 There was little difference in the bootstrap scores for taxonomic assignment between the two

503 methods. Since these bootstrap values are also influenced by the reference database and the

504 reference databases were different for each locus, this comparison should be interpreted with

505 caution. However, the bootstrap scores and branch lengths from the neighbor-joining trees of

506 the mock community sequences were higher in rps10 than in ITS1 (Figure S2; Figure S3),

507 suggesting rps 10 sequences from different taxa are generally more diverged from each other

508 than in similar comparisons for ITS1. Overall, rps10 reference sequences exhibited more

509 polymorphic sites among the most similar inter-species pairwise comparisons than ITS1

510 sequences and were overall more polymorphic.

\section{DISCUSSION}

514 Effective methods for studying microbial communities without the need for culturing and manual identification have greatly increased our understanding of bacterial and fungal ecology, but methods for studying oomycetes are still in need of further development.

517 Metabarcoding is one such method, but it requires a locus and associated primers with

518 particular characteristics, including species-level taxonomic resolution, minimal length

519 variation, the ability to amplify all target organisms, and minimal non-target amplification.

520 The ITS1 locus is a popular choice for oomycete metabarcoding but suffers from limited

521 taxonomic resolution and large variations in length. In addition, currently used primers

522 targeting oomycetes often amplify non-target organisms as well. Here, we propose the $r p s 10$ 
locus as a suitable metabarcoding locus for oomycetes and provide primers that produce an amplicon suitable for use with the Illumina MiSeq. Overall, our results suggest the rps 10 metabarcoding method results in less non-target amplification and has better taxonomic resolution than the ITS1 method tested and is best suited for the preferred ASV-based rather 527 than OTU-based analysis.

Taxonomic specificity

Simulated PCR of rps10 reference sequences showed that all 121 oomycete sequences tested, 532 representing 16 genera, should be amplified by the proposed primers and that non-target taxa, 533 including non-oomycete stramenopiles, should not be amplified (Figure 2). Taxa amplified 534 include species of global and economic concern belonging to the Saprolegniaceae and 535 Peronosporaceae, such as Aphanomyces euteiches, Phytophthora cinnamomi, and Phytophthora infestans. We did not compare the rps10 and ITS1 methods using simulated

537 PCR because ITS1 sequences that included both primer binding sites for many of the species 538 tested in this analysis are not publicly available. This is probably because many ITS1 539 reference sequences are produced with at least one of the primers we used (or primers binding 540 to the same region) and therefore do not include the primer binding sites (Bellemain et al., 541 2010). However, our analysis of MiSeq data from diverse environmental samples suggested 542 that the $\operatorname{rps} 10$ method produced substantially less non-target amplification than the ITS1 543 method in terms of the proportion of non-target reads, ASVs, and OTUs (Figure 3). The 544 unique order of tRNAs flanking the $r p s 10$ gene is likely responsible for this level of 545 amplification specificity (Figure 1). The non-target sequences from ITS1 were nearly all 546 assigned to fungi ( $84.8 \%$ of ASVs, $36.3 \%$ of reads), which conforms to the results of other 
studies (Coince et al., 2013). Although Sapkota and Nicolaisen (2015) suggests increasing annealing temperature as a solution, we still observed much non-target amplification using the ITS1 method at several annealing temperatures (data not shown). Most of the non-target sequences from rps10 had no close match to any sequence in the NCBI nucleotide database ( $12.1 \%$ of ASVs, $0.2 \%$ of reads). These unknown sequences tended to be low abundance,

552 short, and highly dissimilar to rps 10 reference sequences, suggesting they may be erroneous.

553 The minimal non-target amplification of the rps10 method should result in more efficient use 554 of sequencing throughput, making it possible to sequence communities in which oomycetes 555 are rare relative to other organisms like fungi.

557 Sequencing of a mock community using both methods suggest they perform equally well at 558 generating the correct sequences, but that the rps 10 method is better able to generate correct taxonomic classifications. Both methods produced amplicons that perfectly matched 21 of the 24 mock community species and all 24 when a single base pair mismatch was tolerated (Table

5613 3). The ITS1 method produced a slightly greater proportion of ASVs matching mock

562 community members (73.3\% vs. $62.5 \%)$, but for both methods correct ASVs accounted for $563>99 \%$ of the reads. In terms of taxonomic classifications of ASVs, only 1 species out of 24 564 was not detected using the rps 10 method, in contrast to 7 missing with the ITS1 method.

565 Considering that both methods produced sequences matching the same number of mock 566 community members, the higher misclassification rate of the ITS1 method is likely due to 567 insufficient taxonomic resolution. For example, both methods are missing Phytophthora 568 ipomoeae from their taxonomic classifications but do include Phytophthora infestans. Since 569 the amplified sequence for the two species is identical, it is likely that the amplicons for both 570 species were assigned to Phytophthora infestans. This result suggests that the rps 10 region 
571 will produce more accurate taxonomic classifications when applied to real communities of

572 unknown organisms even though the primers for the two methods amplify oomycetes equally

573 well.

Taxonomic resolution

Comparisons of the region predicted to be amplified in reference sequences suggest the rps 10

584 the proportion of species not uniquely identified by rps 10 increased to $16.2 \%$, while that of

585 ITS1 increases to $52.9 \%$. This suggests that most species can be confidently assigned to a

586 species with rps 10, even in the presence of sequencing errors. These results are corroborated

587 by the greater branch lengths observed in neighbor-joining trees of mock community

588 sequences (Figure S3), suggesting the average difference between sequences from different

589 species is greater in rps10. Although rps 10 cannot distinguish all oomycetes species, it has a

590 higher taxonomic resolution than the currently used ITS1 and does not contain the frequent

591 large indels characteristic of ITS1, making it easier to create the multiple sequence alignments

592 needed for many phylogenetic analyses (Figure 5). The superior taxonomic resolution of

$593 \quad$ rps10 will increase the confidence of species classifications, which will be especially helpful

594 in cases where closely related species have different pathological or ecological implications. 


\section{Practical considerations}

The proposed $r p s 10$ method has many attributes that should make it more effective than the

ITS1 method tested, but also a few potential problems to consider. A single, rather than a nested, PCR step should reduce the chance of contamination, sequencing error, and lower the

610 Plasmopara obducens, the longest amplicon in the mock community and the 5th longest in

611 the reference database. Less amplification of non-target organisms should reduce the need to

612 optimize PCR conditions as well as improve amplification efficiency of target organisms due

613 to reduced competition for primers. The greater number of oomycete sequences relative to

614 non-target sequences should allow for more samples for a given sequencing depth. This is a

615 substantial improvement compared to the ITS1 method, in which specificity is very sensitive 616 to the chosen annealing temperature of the PCR (Sapkota \& Nicolaisen, 2015) and even after 617 optimization suffers from non-target amplification of fungi. The greater taxonomic resolution 618 of the rps 10 locus should result in a greater proportion of correct taxonomic classifications, 
which is desirable for monitoring pathogen occurrence. All resources needed to use the rps 10 method for metabarcoding oomycete communities using the Illumina MiSeq, including

621 laboratory protocols and a reference database for taxonomic classification of results, are

622 provided at www.oomycetedb.org.

623 www.oomycetedb.org, along with coxl sequences to confirm species classifications.

\section{Future research}

The global diversity of oomycetes is still largely unknown, with little knowledge of where invasive species come from or their native habitat ranges. This is underlined by results from our environmental samples, where many of the ASVs found had less than $90 \%$ similarity to a reference sequence, meaning ASVs could only be classified at the genus or family level. This is partially because the rps 10 reference database is incomplete and because many, if not most, oomycetes occurring in natural ecosystems have not yet been described. However, there are thousands of oomycete specimens in herbaria around the world that could be leveraged to improve this and other databases. A collaborative effort to sequence rps 10 barcodes from a wider range of oomycetes is in progress and will improve the accuracy and usefulness of this barcode. We encourage the oomycete community to submit rps 10 sequences for inclusion at

638 Oomycetes are an important but relatively understudied group of organisms. Understanding 639 their diversity and distribution will help understand future outbreaks of destructive pathogens 640 like Phytophthora ramorum and Phytophthora infestans and characterize the mostly unknown 641 ecological niches of oomycetes in natural ecosystems. Assuming the species tested here are 642 representative of oomycete diversity, many of the taxonomic classifications of oomycete 
643 microbiomes using ITS1 metabarcoding could be underestimating their true diversity and

644 misclassifying closely related species. Much more research is needed to characterize the

645 natural diversity of oomycetes. Currently, metabarcoding using Illumina sequencing is the

646 most cost-effective technique (Tedersoo et al., 2019). We hope the method presented here will

647 facilitate new insights into oomycete biodiversity and ecology, just as robust methods for

648 metabarcoding of fungi and bacteria have revolutionized our understanding of those

649 organisms in recent decades.

650

651 ACKNOWLEDGEMENTS

652

653 We thank Steve Klosterman, Brett Smith, and Annette Dodge for providing reference DNA.

654 The work was supported in part by: National Science Foundation grant \# DEB-1542681 to

655 NJG, FAJ, and BMT; the USDA-ARS CRIS Project 2072-22000-039-00D to NJG; Project

$6562038-22000-015-00 D$ to FNM; and the USDA-ARS Floriculture Nursery Initiative research

657 programs to NJG.

658

659 AUTHOR CONTRIBUTIONS

660

661 NJG and FNM conceived and designed the study. FNM identified rps10 as a candidate locus

662 for metabarcoding. ZSLF, VJF, MML, FEA, FAJ, and NJG conducted field sampling. VJF,

663 MML, ZSLF, and FEA validated the rps10 locus. ZSLF analyzed sequencing data from

664 metabarcoding. FNM, HDTN, TIB, CR, and NJG provided reference DNA or sequences for

665 the reference database. ZSLF, VJF, MML, and NJG developed and maintain the oomycete- 
666 DB database and website. BMT, FAJ, and NJG were responsible for overall project design

667 and obtained funding. All authors edited and approved the final version of the manuscript.

668

\section{REFERENCES}

670

Altschul, S. F., Gish, W., Miller, W., Myers, E. W., \& Lipman, D. J. (1990). Basic local alignment search tool. Journal of Molecular Biology, 215(3), 403-410.

Andersson, S. G. E., Zomorodipour, A., Andersson, J. O., Sicheritz-Pontén, T., Alsmark, U. doi: $10.1038 / 24094$

Augspurger, C. K., \& Wilkinson, H. T. (2007). Host specificity of pathogenic Pythium species: Implications for tree species diversity. Biotropica, 39(6), 702-708. doi:

Baldauf, S. L., Roger, A. J., Wenk-Siefert, I., \& Doolittle, W. F. (2000). A kingdom-level 290(5493), 972-977.

684

Brasier, C., \& Webber, J. (2010). Plant pathology: Sudden larch death. Nature, 466(7308), 824-825. doi: $10.1038 / 466824 a$

Bukin, Yu. S., Galachyants, Yu. P., Morozov, I. V., Bukin, S. V., Zakharenko, A. S., \& Zemskaya, T. I. (2019). The effect of 16S rRNA region choice on bacterial community metabarcoding results. Scientific Data, 6(1), 190007. doi: 
691

692

693

694

695

696

697

698

699

700

701

702

703

704

705

706

707

708

709

710

711

712

713

714

cinnamomi, one of the world's worst plant pathogens. Global Change Biology, 23(4),

$$
\text { 1661-1674. doi: 10.1111/gcb.13492 }
$$

Cahill, D. M., Rookes, J. E., Wilson, B. A., Gibson, L., \& McDougall, K. L. (2008).

Phytophthora cinnamomi and Australia's biodiversity: Impacts, predictions and progress towards control. Australian Journal of Botany, 56(4), 279. doi: 10.1071/BT07159

Callahan, B. J., McMurdie, P. J., \& Holmes, S. P. (2017). Exact sequence variants should replace operational taxonomic units in marker-gene data analysis. The ISME Journal, $11(12), 2639$.

Callahan, B. J., McMurdie, P. J., Rosen, M. J., Han, A. W., Johnson, A. J. A., \& Holmes, S. P. (2016). DADA2: High-resolution sample inference from Illumina amplicon data. Nature Methods, 13(7), 581-583. doi: 10.1038/nmeth.3869

Chen, W., Zhang, C. K., Cheng, Y., Zhang, S., \& Zhao, H. (2013). A comparison of methods for clustering 16S rRNA sequences into OTUs. PloS One, 8(8), e70837.

Choi, Y.-J., Beakes, G., Glockling, S., Kruse, J., Nam, B., Nigrelli, L., ... Thines, M. (2015). Towards a universal barcode of oomycetes-A comparison of the cox 1 and cox 2 loci. Molecular Ecology Resources, 15(6), 1275-1288. doi: 10.1111/1755-0998.12398

Coince, A., Caël, O., Bach, C., Lengellé, J., Cruaud, C., Gavory, F., ... Buée, M. (2013). Below-ground fine-scale distribution and soil versus fine root detection of fungal and soil oomycete communities in a French beech forest. Fungal Ecology, 6(3), 223-235. doi: 10.1016/j.funeco.2013.01.002

Cooke, D. E. L., Drenth, A., Duncan, J. M., Wagels, G., \& Brasier, C. M. (2000). A molecular phylogeny of Phytophthora and related Oomycetes. Fungal Genetics and Biology, 30, 17-32. doi: 10.1006/fgbi.2000.1202 
Cristescu, M. E. (2014). From barcoding single individuals to metabarcoding biological communities: Towards an integrative approach to the study of global biodiversity. Trends in Ecology \& Evolution, 29(10), 566-571. doi: 10.1016/j.tree.2014.08.001

Esmaeili Taheri, A., Chatterton, S., Gossen, B., \& McLaren, D. (2017). Degenerate ITS7 primer enhances oomycete community coverage and PCR sensitivity to Aphanomyces species, economically important plant pathogens. Canadian Journal of Microbiology, 63(9), 769-779.

Fiore-Donno, A. M., \& Bonkowski, M. (2021). Different community compositions between obligate and facultative oomycete plant parasites in a landscape-scale metabarcoding survey. Biology and Fertility of Soils, 57(2), 245-256.

Foster, Z. S. L., Chamberlain, S., \& Grünwald, N. J. (2018). Taxa: An R package implementing data standards and methods for taxonomic data. F1000Research, 7, 272. doi: 10.12688/f1000research.14013.2

Foster, Z. S. L., Sharpton, T. J., \& Grünwald, N. J. (2017). Metacoder: An R package for visualization and manipulation of community taxonomic diversity data. PLOS Computational Biology, 13(2), e1005404. doi: 10.1371/journal.pcbi.1005404

Foster, Z. S. L., Weiland, J. E., Scagel, C. F., \& Grünwald, N. J. (2020). The composition of the fungal and oomycete microbiome of Rhododendron roots under varying growth conditions, nurseries, and cultivars. Phytobiomes Journal, PBIOMES-09-19-0. doi: 10.1094/PBIOMES-09-19-0052-R

Fry, W. (2008). Phytophthora infestans: The plant (and R gene) destroyer. Molecular Plant Pathology, 9(3), 385-402. doi: 10.1111/j.1364-3703.2007.00465.x 
Gaulin, E., Jacquet, C., Bottin, A., \& Dumas, B. (2007). Root rot disease of legumes caused by Aphanomyces euteiches. Molecular Plant Pathology, 8(5), 539-548. doi:

Grünwald, N. J., Goss, E. M., \& Press, C. M. (2008). Phytophthora ramorum: A pathogen with a remarkably wide host range causing sudden oak death on oaks and ramorum blight on woody ornamentals. Molecular Plant Pathology, 9(6), 729-740. doi:

Grünwald, N. J., LeBoldus, J. M., \& Hamelin, R. C. (2019). Ecology and evolution of the sudden oak death pathogen Phytophthora ramorum. Annual Review of Phytopathology, 57(1), 301-321. doi: 10.1146/annurev-phyto-082718-100117

Hansen, E. M., Reeser, P. W., \& Sutton, W. (2012). Phytophthora beyond agriculture. Annual Review of Phytopathology, 50, 359-378.

Katoh, K. (2005). MAFFT version 5: Improvement in accuracy of multiple sequence alignment. Nucleic Acids Research, 33(2), 511-518. doi: 10.1093/nar/gki198

Kõljalg, U., Larsson, K.-H., Abarenkov, K., Nilsson, R. H., Alexander, I. J., Eberhardt, U., ... others. (2005). UNITE: a database providing web-based methods for the molecular identification of ectomycorrhizal fungi. New Phytologist, 166(3), 1063-1068. Pérez-Sierra, A. (2021). Diversity of Phytophthora Species Detected in Disturbed and Undisturbed British Soils Using High-Throughput Sequencing Targeting ITS rRNA and COI mtDNA Regions. Forests, 12(2), 229. and validation of Oomycetes metabarcoding primers for Phytophthora high 
throughput sequencing. Journal of Plant Pathology, 101(3), 743-748. doi:

Martin, F. N., Bensasson, D., Tyler, B. M., \& Boore, J. L. (2007). Mitochondrial genome sequences and comparative genomics of Phytophthora ramorum and P. sojae. Current Genetics, 51(5), 285-296. doi: 10.1007/s00294-007-0121-6

Martin, F. N., Blair, J. E., \& Coffey, M. D. (2014). A combined mitochondrial and nuclear multilocus phylogeny of the genus Phytophthora $</ \mathrm{i}><\mathrm{i}>$. Fungal Genetics and Biology, 66, 19-32. doi: 10.1016/j.fgb.2014.02.006

Martin, F. N., \& Loper, J. E. (1999). Soilborne plant diseases caused by Pythium spp.: Ecology, epidemiology, and prospects for biological control. Critical Reviews in Plant Sciences, 18(2), 111-181. doi: 10.1080/07352689991309216

Martin, M. (2011). Cutadapt removes adapter sequences from high-throughput sequencing

773 reads. EMBnet.Journal, 17(1), 10. doi: 10.14806/ej.17.1.200

Nichols, R. V., Vollmers, C., Newsom, L. A., Wang, Y., Heintzman, P. D., Leighton, M., ... Shapiro, B. (2018). Minimizing polymerase biases in metabarcoding. Molecular Ecology Resources, 18(5), 927-939. doi: 10.1111/1755-0998.12895

Nilsson, R. H., Anslan, S., Bahram, M., Wurzbacher, C., Baldrian, P., \& Tedersoo, L. (2019). Mycobiome diversity: High-throughput sequencing and identification of fungi. Nature Reviews Microbiology, 17(2), 95-109. doi: 10.1038/s41579-018-0116-y

Nilsson, R. H., Ryberg, M., Kristiansson, E., Abarenkov, K., Larsson, K.-H., \& Kõljalg, U. (2006). Taxonomic reliability of DNA sequences in public sequence databases: A fungal perspective. PLoS ONE, 1(1), e59. doi: 10.1371/journal.pone.0000059

Owczarzy, R., Tataurov, A. V., Wu, Y., Manthey, J. A., McQuisten, K. A., Almabrazi, H. G., ... Peek, A. S. (2008). IDT SciTools: A suite for analysis and design of nucleic acid 
oligomers. Nucleic Acids Research, 36(Web Server), W163-W169. doi:

Pages, H., Aboyoun, P., Gentleman, R., \& DebRoy, S. (2017). Biostrings: String objects representing biological sequences, and matching algorithms. $R$ package version 2.36.4. R package version 2.36.4.

Park, J., Park, B., Veeraraghavan, N., Jung, K., Lee, Y.-H., Blair, J. E., ... others. (2008). monitoring of Phytophthora. Plant Disease, 92(6), 966-972.

792

Phillips, A. J., Anderson, V. L., Robertson, E. J., Secombes, C. J., \& van West, P. (2008). New insights into animal pathogenic oomycetes. Trends in Microbiology, 16(1), 1319. doi: 10.1016/j.tim.2007.10.013

Redekar, N. R., Eberhart, J. L., \& Parke, J. L. (2019). Diversity of Phytophthora, Pythium, and Phytopythium species in recycled irrigation water in a container nursery. Phytobiomes Journal, 3(1), 31-45. doi: 10.1094/PBIOMES-10-18-0043-R

Riddell, C. E., Frederickson-Matika, D., Armstrong, A. C., Elliot, M., Forster, J., Hedley, P. E., ... others. (2019). Metabarcoding reveals a high diversity of woody host-associated Phytophthora spp. In soils at public gardens and amenity woodlands in Britain. PeerJ, 7, e6931.

Riit, T., Tedersoo, L., Drenkhan, R., Runno-Paurson, E., Kokko, H., \& Anslan, S. (2016). Oomycete-specific ITS primers for identification and metabarcoding. MycoKeys, 14, 17-30. doi: 10.3897/mycokeys.14.9244

Robideau, G. P., De COCK, A. W. A. M., Coffey, M. D., Voglmayr, H., Brouwer, H., Bala, K., ... André Lévesque, C. (2011). DNA barcoding of oomycetes with cytochrome c 
807

808

809

810

oxidase subunit I and internal transcribed spacer. Molecular Ecology Resources, 11(6), 1002-1011. doi: 10.1111/j.1755-0998.2011.03041.x

Rognes, T., Flouri, T., Nichols, B., Quince, C., \& Mahé, F. (2016). VSEARCH: A versatile open source tool for metagenomics. PeerJ, 4, e2584. doi: 10.7717/peerj.2584

Salcedo, A. F., Purayannur, S., Standish, J. R., Miles, T., Thiessen, L., \& Quesada-Ocampo, L. M. (2021). Fantastic Downy Mildew Pathogens and How to Find Them: Advances in Detection and Diagnostics. Plants, 10(3), 435.

SantaLucia, J. (1998). A unified view of polymer, dumbbell, and oligonucleotide DNA nearest-neighbor thermodynamics. Proceedings of the National Academy of Sciences, 95(4), 1460-1465. doi: 10.1073/pnas.95.4.1460

Sapkota, R., \& Nicolaisen, M. (2015). An improved high throughput sequencing method for studying oomycete communities. Journal of Microbiological Methods, 110, 33-39. doi: 10.1016/j.mimet.2015.01.013

Sapp, M., Lewis, E., Moss, S., Barrett, B., Kirk, S., Elphinstone, J. G., \& Denman, S. (2016). Metabarcoding of bacteria associated with the Acute Oak Decline syndrome in England. Forests, 7(5), 95.

Schappe, T., Albornoz, F. E., Turner, B. L., Neat, A., Condit, R., \& Jones, F. A. (2017). The role of soil chemistry and plant neighbourhoods in structuring fungal communities in three Panamanian rainforests. Journal of Ecology, 105(3), 569-579. doi: $10.1111 / 1365-2745.12752$

Schoch, C. L., Seifert, K. A., Huhndorf, S., Robert, V., Spouge, J. L., Levesque, C. A., ... Schindel, D. (2012). Nuclear ribosomal internal transcribed spacer (ITS) region as a universal DNA barcode marker for Fungi. Proceedings of the National Academy of Sciences, 109(16), 6241-6246. doi: 10.1073/pnas.1117018109 
831 Sievers, F., Wilm, A., Dineen, D., Gibson, T. J., Karplus, K., Li, W., ... Higgins, D. G.

832

833

834

835

836

837

838

839

840

841

842

843

844

845

846

847

848

849

850

851

852

853

(2011). Fast, scalable generation of high-quality protein multiple sequence alignments using Clustal Omega. Molecular Systems Biology, 7(1), 539. doi:

10.1038/msb.2011.75

Spring, O., Gomez-Zeledon, J., Hadziabdic, D., Trigiano, R. N., Thines, M., \& Lebeda, A. (2018). Biological characteristics and assessment of virulence diversity in pathosystems of economically important biotrophic oomycetes. Critical Reviews in Plant Sciences, 37(6), 439-495.

Tedersoo, L., Drenkhan, R., Anslan, S., Morales-Rodriguez, C., \& Cleary, M. (2019). Highthroughput identification and diagnostics of pathogens and pests: Overview and practical recommendations. Molecular Ecology Resources, 19(1), 47-76. doi: $10.1111 / 1755-0998.12959$

Tringe, S. G., \& Hugenholtz, P. (2008). A renaissance for the pioneering 16S rRNA gene. Current Opinion in Microbiology, 11(5), 442-446. doi: 10.1016/j.mib.2008.09.011

van West, P. (2006). Saprolegnia parasitica, an oomycete pathogen with a fishy appetite: New challenges for an old problem. Mycologist, 20(3), 99-104. doi: 10.1016/j.mycol.2006.06.004

Wang, Q., Garrity, G. M., Tiedje, J. M., \& Cole, J. R. (2007). Naive Bayesian classifier for rapid assignment of rRNA sequences into the new bacterial taxonomy. Applied and Environmental Microbiology, 73(16), 5261-5267. doi: 10.1128/AEM.00062-07

White, T. J., Bruns, T., Lee, S., \& Taylor, J. (1990). Amplification and direct sequencing of fungal ribosomal RNA genes for phylogenetics. In PCR Protocols (pp. 315-322). Elsevier. doi: 10.1016/B978-0-12-372180-8.50042-1 
854 Wills, R. T. (1993). The ecological impact of Phytophthora cinnamomi in the Stirling Range

Xie, Y., Allaire, J. J., \& Grolemund, G. (2019). R Markdown: The definitive guide. Retrieved from http://search.ebscohost.com/login.aspx $?$ direct $=$ true $\&$ scope $=$ site $\& d b=$ nlebk $\& \mathrm{db}=$ nlabk $\& \mathrm{AN}=1863232$

Yoon, H. S., Hackett, J. D., Pinto, G., \& Bhattacharya, D. (2002). The single, ancient origin of chromist plastids. Proceedings of the National Academy of Sciences, 99(24), 15507-15512. doi: 10.1073/pnas.242379899

864 Yuan, X., Feng, C., Zhang, Z., \& Zhang, C. (2017). Complete mitochondrial genome of Phytophthora nicotianae and identification of molecular markers for the oomycetes. 


\section{DATA ACCESSIBILITY}

869

870 All rps10 sequences were submitted to GenBank (MZ365320-MZ365440) and metabarcoding

871 data were submitted to the SRA (Bioproject PRJNA699663; Accessions SRR13658632

872 - SRR13658679). A curated rps10 database for taxonomic classification is publicly available

873 at www.oomyceteDB.org for downloading. We encourage submission of new species to the

874 database to improve this resource for the community (see instructions online). Reproducible

875 code for the analysis presented here is available at

876 https://github.com/grunwaldlab/rps10_barcode. 
879 Table 1. List of primers used for rps 10 metabarcoding and the reference database entries.

\begin{tabular}{|c|c|c|}
\hline Primer name & rps10 metabarcoding primer sequence $\left(5^{\prime}-3^{\prime}\right)^{\dagger}$ & Reference \\
\hline rps10_F1 & $\begin{array}{l}\text { TCGTCGGCAGCGTCAGATGTGTATAAGAGACAG } \\
\text { GTTGGTTAGAGYAAAAGACT }\end{array}$ & This study \\
\hline rps10_F2 & $\begin{array}{l}\text { TCGTCGGCAGCGTCAGATGTGTATAAGAGACAG } \\
\text { GTTGGTTAGAGTAGAAGACT }\end{array}$ & This study \\
\hline rps10_R1 & $\begin{array}{l}\text { GTCTCGTGGGCTCGGAGATGTGTATAAGAGACAG } \\
\text { ATGCTTAGAAAGATTTGAACT }\end{array}$ & This study \\
\hline rps10_R2 & $\begin{array}{l}\text { GTCTCGTGGGCTCGGAGATGTGTATAAGAGACAG } \\
\text { ATACTTAGAAAGATTTGAACT }\end{array}$ & This study \\
\hline rps10_R3 & $\begin{array}{l}\text { GTCTCGTGGGCTCGGAGATGTGTATAAGAGACAG } \\
\text { ATGCTTAGAAAGACTTGAACT }\end{array}$ & This study \\
\hline rps10_R4 & $\begin{array}{l}\text { GTCTCGTGGGCTCGGAGATGTGTATAAGAGACAG } \\
\text { ATGCTTAGAAAGACTCGAACT }\end{array}$ & This study \\
\hline rps10_R5 & $\begin{array}{l}\text { GTCTCGTGGGCTCGGAGATGTGTATAAGAGACAG } \\
\text { ATGCCTAGAAAGACTCGAACT }\end{array}$ & This study \\
\hline rps10_R6 & $\begin{array}{l}\text { GTCTCGTGGGCTCGGAGATGTGTATAAGAGACAG } \\
\text { ATGTTTAGAAAGATTCGAACT }\end{array}$ & This study \\
\hline
\end{tabular}




\begin{tabular}{lcl} 
rps10_R7 & $\begin{array}{l}\text { GTCTCGTGGGCTCGGAGATGTTATAAGAGACAG } \\
\text { ATGCTTAGAAAGATTCGAACT }\end{array}$ & This study \\
\hline Primer name & rps10 database primer sequence (5'-3') & Reference \\
\hline rps10_DB_F $\mathbf{5}^{*}$ & GTTGGTTAGAGYARAAGACT & This study \\
rps10_DB_R & RTAYACTCTAACCAACTGAGT & This study \\
\hline & ITS1 Primer sequence (5'-3') & \\
\hline ITS6 & GAAGGTGAAGTCGTAACAAGG & (Cooke et al., 2000) \\
ITS4 & TCCTCCGCTTATTGATATGC & (White, Bruns, Lee, \\
& & \& Taylor, 1990) \\
ITS7 & AGCGTTCTTCATCGATGTGC & (Cooke et al., 2000) \\
\hline
\end{tabular}

$881 \dagger^{\prime}$ ' Illumina overhang adapter sequences are italicized. Locus-specific sequences are regular font. Forward and reverse primers for $r p s$ 10 882 metabarcoding are labelled with letters $\mathrm{F}$ and $\mathrm{R}$ in the primer name, respectively.

$883 \$$ Primers for generating amplicons that include more of the tRNA genes flanking the $r p s 10$ gene for database reference sequences are from

884 Martin et al. (2014) modified with degenerate bases and renamed. 
885 Table 2. Overview of the number of sequences currently available in the rps 10 barcode

886 database.

\begin{tabular}{lr}
\hline Genus & Number of sequences \\
\hline Achlya & 1 \\
Albugo & 1 \\
Aphanomyces & 13 \\
Bremia & 3 \\
Halophytophthora & 9 \\
Hyaloperonospora & 1 \\
Lagena & 1 \\
Lagenidium & 2 \\
Perofascia & 1 \\
Peronosclerospora & 4 \\
Peronospora & 73 \\
Phytophthora & 509 \\
Phytopythium & 25 \\
Plasmopara & 5 \\
Pseudoperonospora & 3 \\
Pythium & $\mathbf{8 8 6}$ \\
Salisapilia & 226 \\
Saprolegnia & 4 \\
Sclerospora & 3 \\
Thraustotheca & 1 \\
\hline Total & 1 \\
\hline & \\
\hline
\end{tabular}


888 Table 3. Mock community inference statistics based on proportions of correct taxonomic

889 classifications and proportions of correct sequences.

\begin{tabular}{lll|lll|llll}
\hline \multicolumn{3}{l}{ Total counts } & \multicolumn{2}{l}{ Correct taxonomy } & \multicolumn{3}{l}{ Correct sequence } \\
\hline Locus & ASVs & OTUs & MC Species $^{\dagger}$ & ASVs $^{\ddagger}$ & Reads $^{\S}$ & MC Species $^{\dagger}$ & ASVs $^{\ddagger}$ & Reads $^{\S}$ \\
$\operatorname{rps10}$ & 40 & 46 & $0.958(23)$ & 0.950 & $>0.999$ & $0.875(21)$ & 0.625 & 0.993 \\
ITS1 & 45 & 30 & $0.708(17)$ & 0.756 & 0.545 & $0.875(21)$ & 0.733 & 0.992 \\
\hline
\end{tabular}

$890 \dagger$ Proportion of mock community species detected; numbers in brackets are species observed.

$891 \ddagger$ Proportion of ASVs matching a mock community species.

$892 \S$ Proportion of reads matching a mock community species. 
893

\section{FIGURES}

894

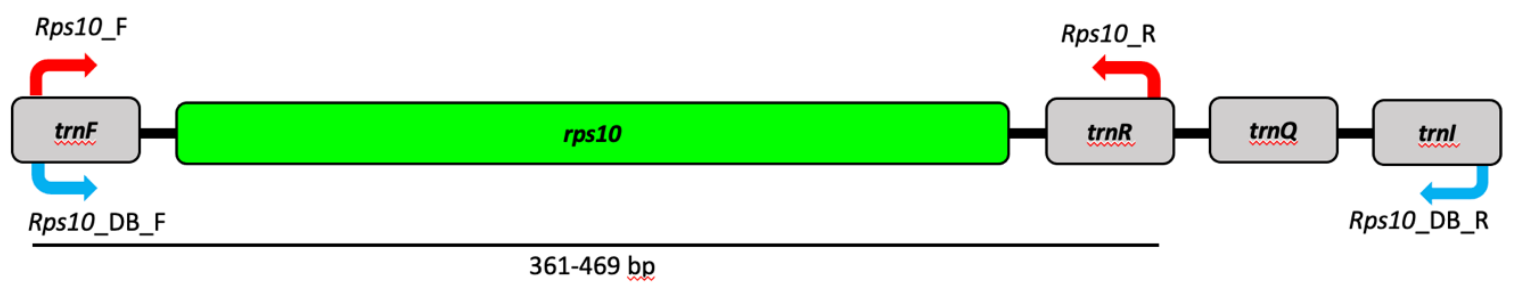

895

896 Figure 1. The location of the 40S ribosomal protein S10 (rps 10) locus in the mitochondrial

897 genome of oomycetes. The rps10 locus is flanked by several tRNA genes (F. N. Martin,

898 Bensasson, Tyler, \& Boore, 2007). Primers shown in blue are for amplification of the

899 sequences found in the reference database, and primers shown in red are for metabarcoding.

900 More details on the two sets of primers are provided in Table 1. 


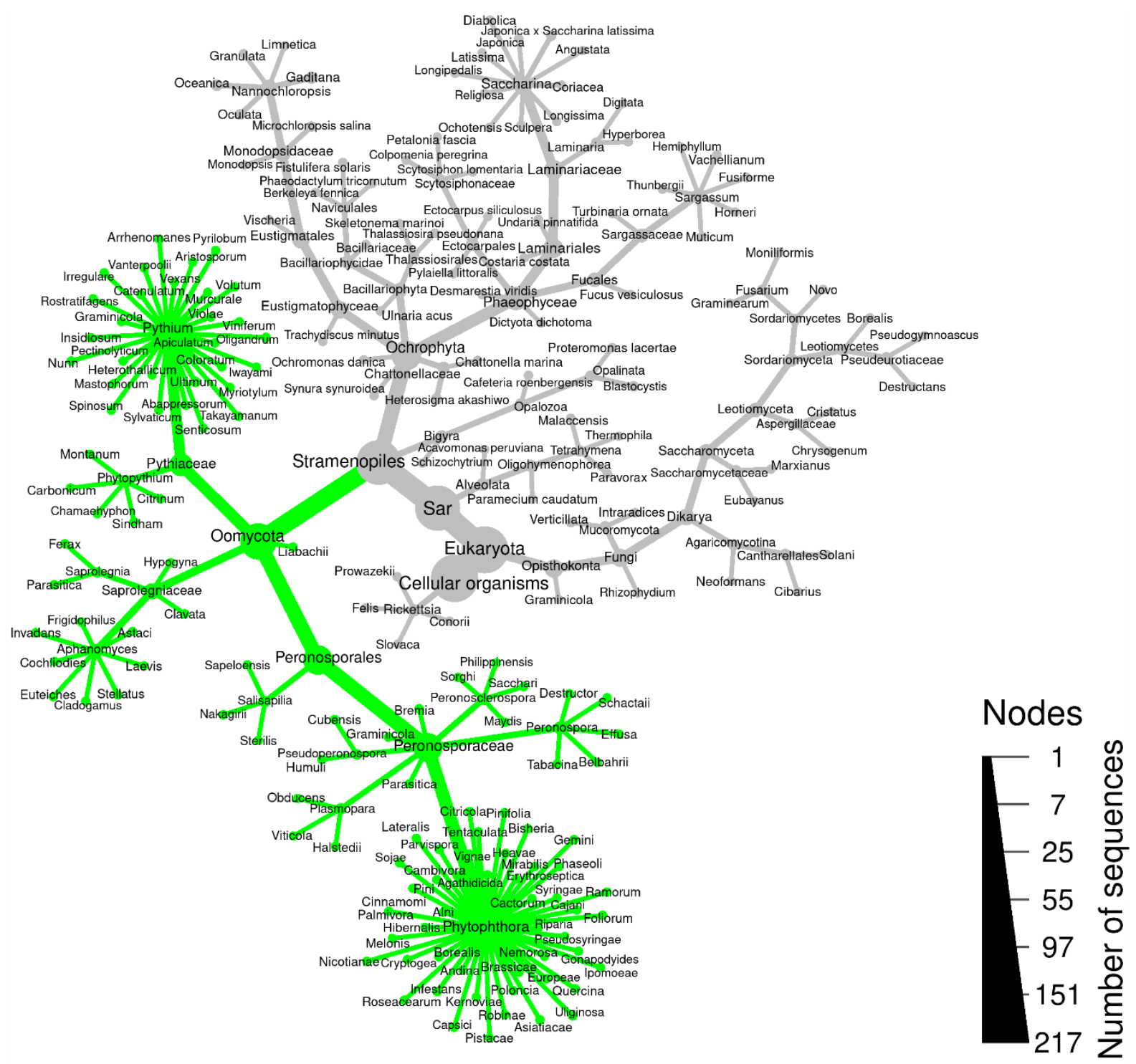

901

902 Figure 2. Heat tree showing predicted amplification (green) of the rps 10 metabarcoding

903 primers. Taxa in green are predicted to be amplified using simulated PCR. The analysis

904 included fungi and related Stramenopiles to demonstrate specificity. Rickettsia were also

905 included since their genomes resemble mitochondrial genomes. The size of branches and

906 nodes is relative to the number of sequences represented by each taxon. 
bioRxiv preprint doi: https//doi.org/10.1101/2021.09.22.460084 this version posted September 22, 2021. The copyright holder for this preprint (which was not certified by peer review) is the author/funder, who has granted bioRxiv a license to display the preprint in perpetuity. It is made available under aCC-BY-ND 4.0 International license.

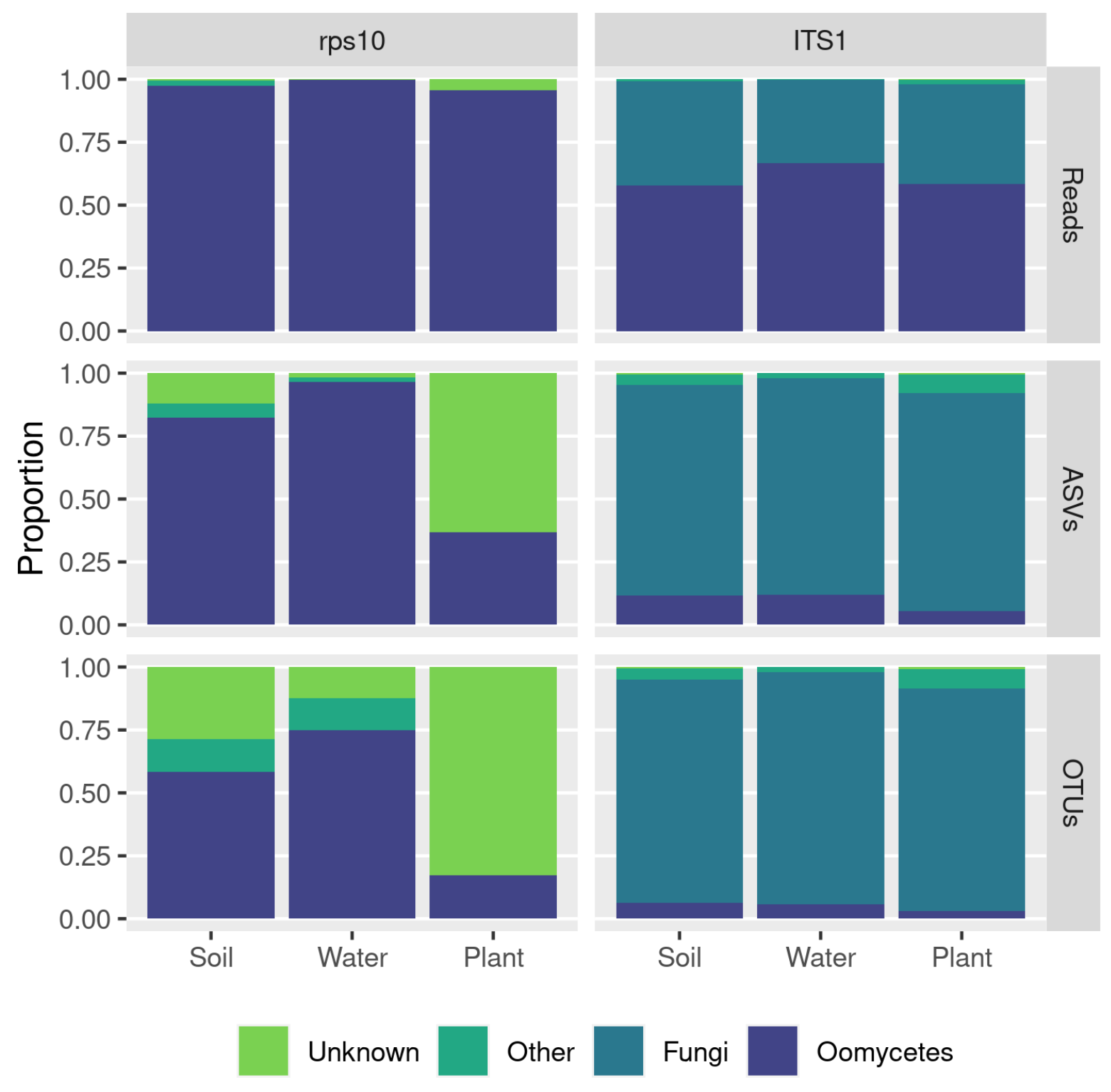

908 Figure 3. Taxonomic specificity of amplification using the rps 10 and ITS1 methods based on

909 environmental samples. Counts of read, amplified sequence variants (ASVs), and operational

910 taxonomic units (OTUs) from a variety of environmental samples grouped into soil, water,

911 and plant tissue samples are shown. Here, ASV sequences were given a taxonomic

912 assignment based on BLAST searches against the NCBI nucleotide database. 

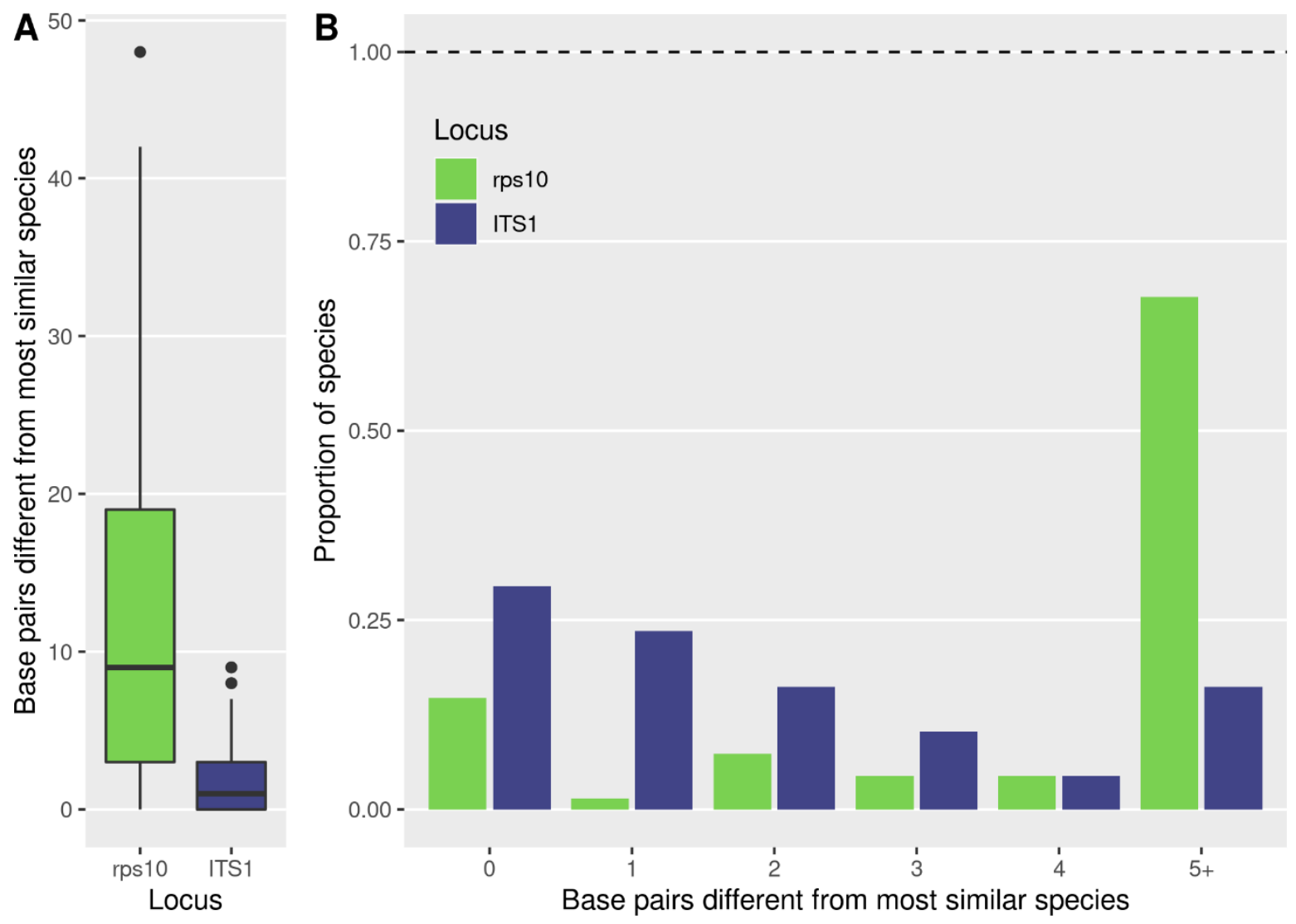

914 Figure 4. Taxonomic resolution of the rps10 (green) and ITS1 (blue) barcodes. We evaluated

915 the number of base pair differences to the most similar species based on pairwise alignments

916 of predicted amplicons. (A) Distribution of the number of base pair differences between the

917 most similar species. (B) Number of polymorphic sites differentiating the most similar

918 species. Zero differences for a species mean there is at least one other species predicted to

919 have an identical amplicon sequence. Only sequences for species present in both databases are 920 included. 
bioRxiv preprint doi: https://doi.org/10.1101/2021.09.22.460084; this version posted September 22, 2021. The copyright holder for this preprint (which was not certified by peer review) is the author/funder, who has granted bioRxiv a license to display the preprint in perpetuity. It is made available under aCC-BY-ND 4.0 International license.
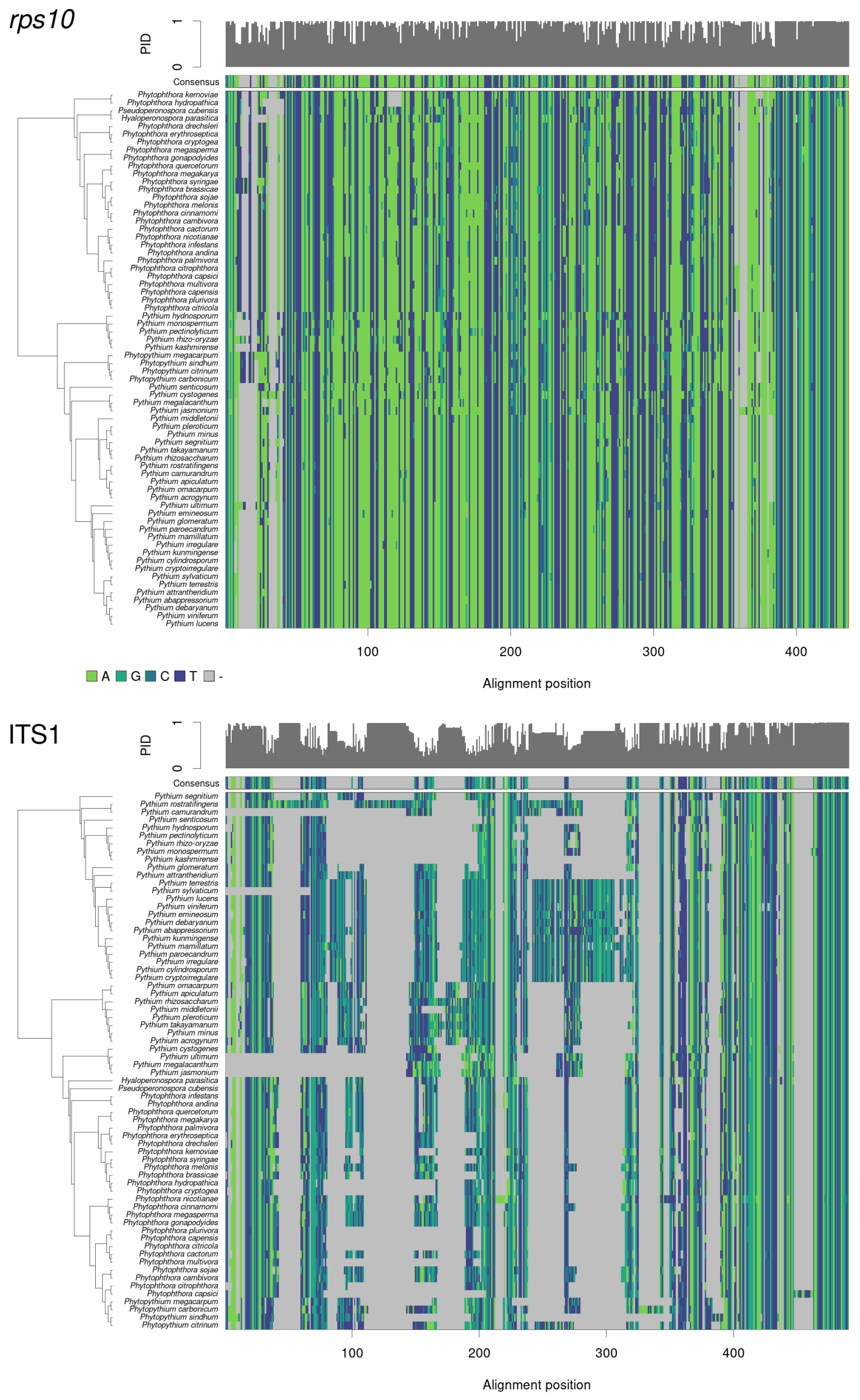
922 Figure 5. Multiple sequence alignments of the region predicted to be amplified by the rps 10

923 or ITS1 method, not including the primer binding sites. The sequences used represent the

924 subset of species present in both reference databases. The sequences are ordered vertically

925 based on a neighbor-joining tree. Along the top of each alignment is a bar chart representing

926 the proportion of sequences matching the consensus sequence at each alignment position.

927 Gray blocks are gaps in the alignment. 


\section{Supporting Information}

Table S1. List of species included in the mock community. Table includes final DNA concentration in the mock community, isolate name, growth medium, and DNA extraction method.

\begin{tabular}{|c|c|c|c|c|}
\hline Species & $\begin{array}{c}\text { Concentration } \\
(\mathrm{ng} / \mu \mathrm{L})\end{array}$ & Isolate & Growth Medium & Extraction Method \\
\hline Aphanomyces euteiches & 2 & MER4 & CMA & MPBio FastDNA \\
\hline Peronospora effusa & 2 & $\mathrm{n} / \mathrm{a}$ & Spinacia oleracea & Macherey-Nagel NucleoSpin Plant II \\
\hline Peronospora schachtii & 2 & $\mathrm{n} / \mathrm{a}$ & Spinacia oleracea & Macherey-Nagel NucleoSpin Plant II \\
\hline Phytopythium citrinum & 2 & $\begin{array}{l}\text { CBS119171 } \\
\text { (Type) }\end{array}$ & & \\
\hline Phytophthora cinnamomi & 2 & Cin-TJ-130 & PARP media & Dneasy Plant mini kit \\
\hline Phytophthora citrophthora & 2 & 6400-PR-16 & PARP media & Dneasy Plant mini kit \\
\hline Phytophthora himalsilva & 2 & $531-\mathrm{P}-13$ & PARP media & Dneasy Plant mini kit \\
\hline Phytophthora hydrogena & 2 & 46A3 (type) & & \\
\hline Phytophthora infestans & 2 & 1826-PI-07 & & \\
\hline Phytophthora ipomoeae & 2 & 1990-PI-07 & & \\
\hline Phytophthora kernoviae & 2 & $55-\mathrm{PK}-05$ & PARP media & Dneasy Plant mini kit \\
\hline Phytophthora pluvialis & 0.7 & $\begin{array}{l}\text { 14Mar- } \\
\text { EMR }\end{array}$ & PARP media & Dneasy Plant mini kit \\
\hline
\end{tabular}




\begin{tabular}{lclll} 
& \multicolumn{5}{c}{ P10334 } & & \\
Phytophthora quercina & 2 & WPC & & \\
Phytophthora ramorum & 2 & $6579-P R-17$ & PARP media & Dneasy Plant mini kit \\
Phytophthora rosacearum & 2 & 52 (type) & & \\
Plasmopara halstedii & 2 & RDM15 & Rudbeckia sp. & Qiagen DNeasy Plant Mini Kit \\
& & & Impatiens & \\
Plasmopara obducens & 2 & MD15.4 & walleriana & Dneasy Plant mini kit \\
$\begin{array}{l}\text { Pseudoperonospora cubensis } \\
\text { Pythium apiculatum }\end{array}$ & 2 & MSU-1 & Cucumis sativus & Ahmed et al. 2009 protocol \\
$\begin{array}{l}\text { Pythium dissotocum } \\
\text { Pythium irregulare }\end{array}$ & 2 & CBS120945 & & \\
Pythium oligandrum & 2 & ML74 & PARP media & Dneasy Plant mini kit \\
Pythium undulatum & 2 & S-F-63 & PARP media & Dneasy Plant mini kit \\
Saprolegnia diclina & 2 & Py1988-14 & & \\
\hline
\end{tabular}




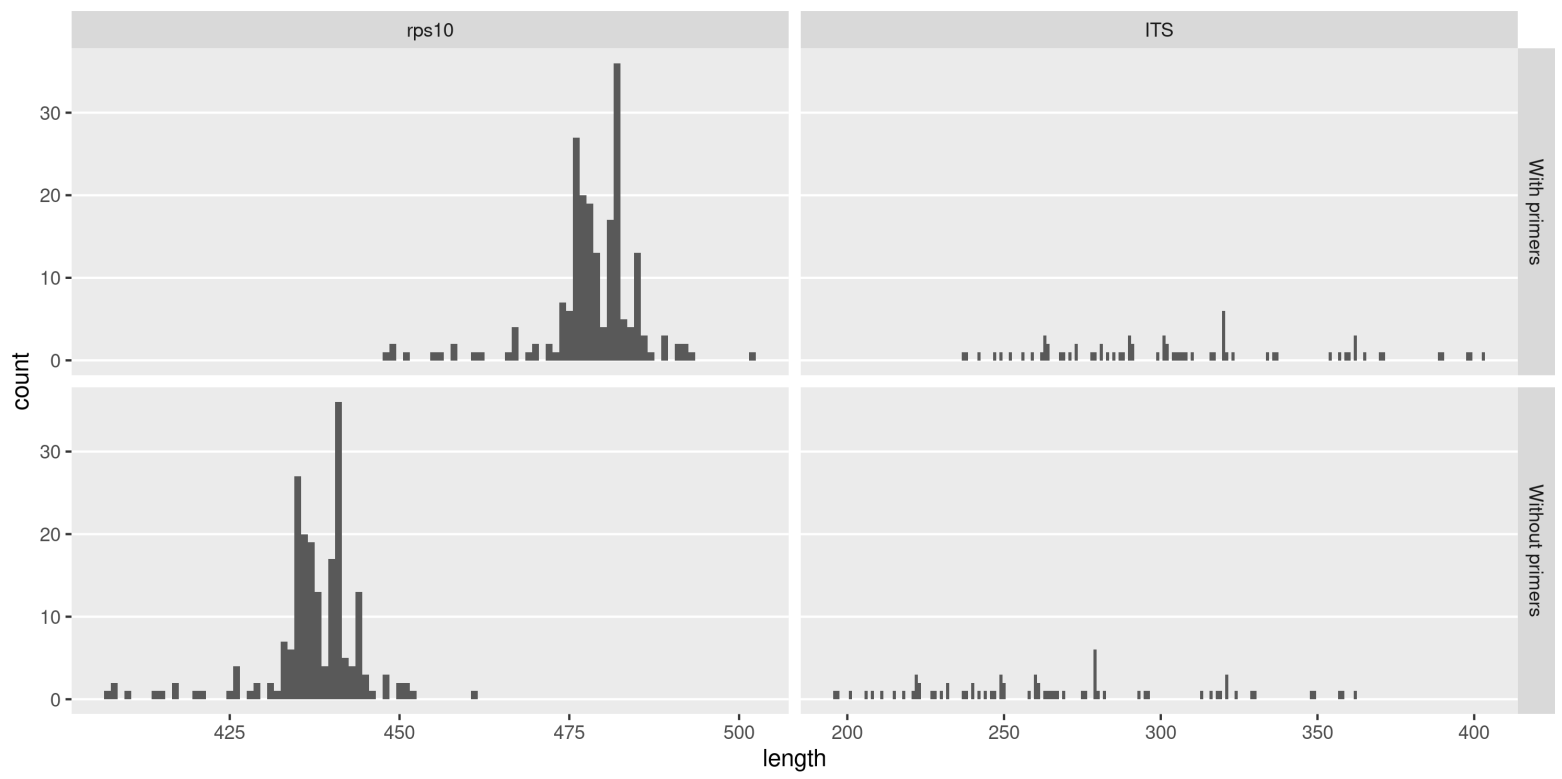

934 Figure S1. The distribution of amplicon lengths predicted to be produced by the rps 10 and

935 ITS1 methods tested, with and without the primer sites (without the Illumina adapters), based

936 on reference database sequences. Only species present in both databases are included. 


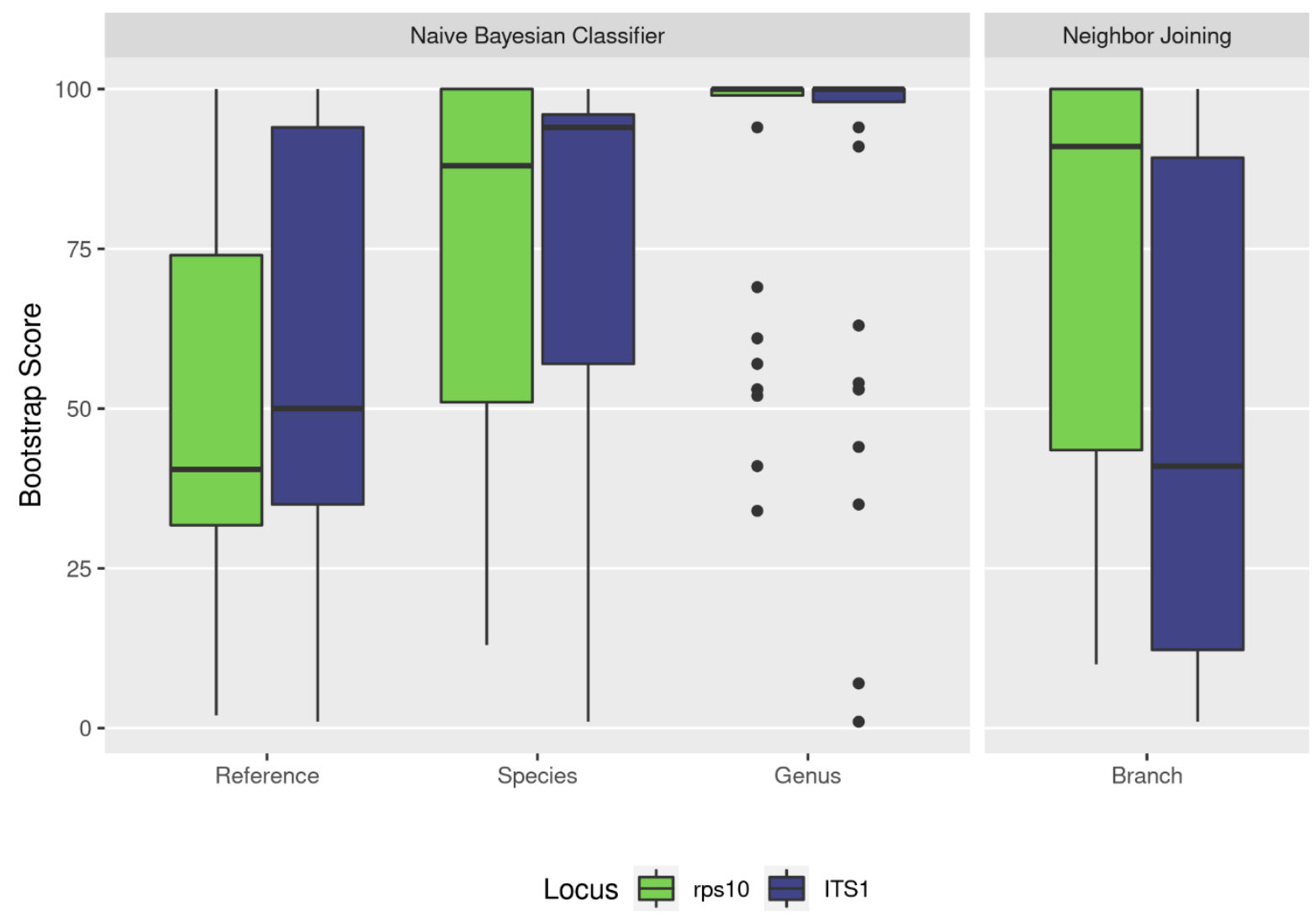

938 Figure S2. The distribution of bootstrap scores for the taxonomic assignment of amplified

939 sequence variants (ASVs) in the mock community for the rps10 and ITS1 loci. The RDP

940 Naive Bayesian Classifier "Reference", "Species", and "Genus" scores refer to the ability to

941 consistently assign ASVs to a particular reference sequence, species, or genus respectively

942 when the data is resampled. The neighbor joining tree scores quantify how consistent the

943 branching pattern of the resulting tree is when the data is resampled. 
A. ITS1

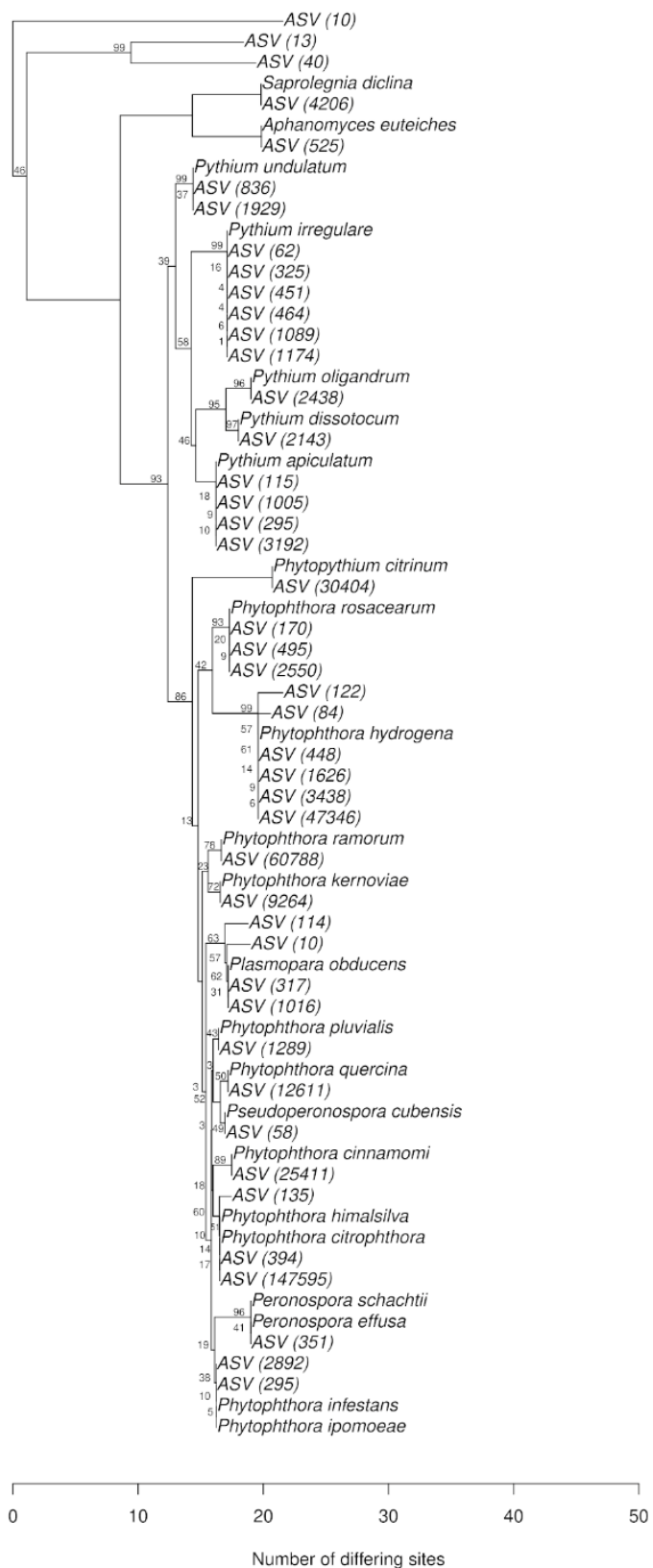

B. RPS10

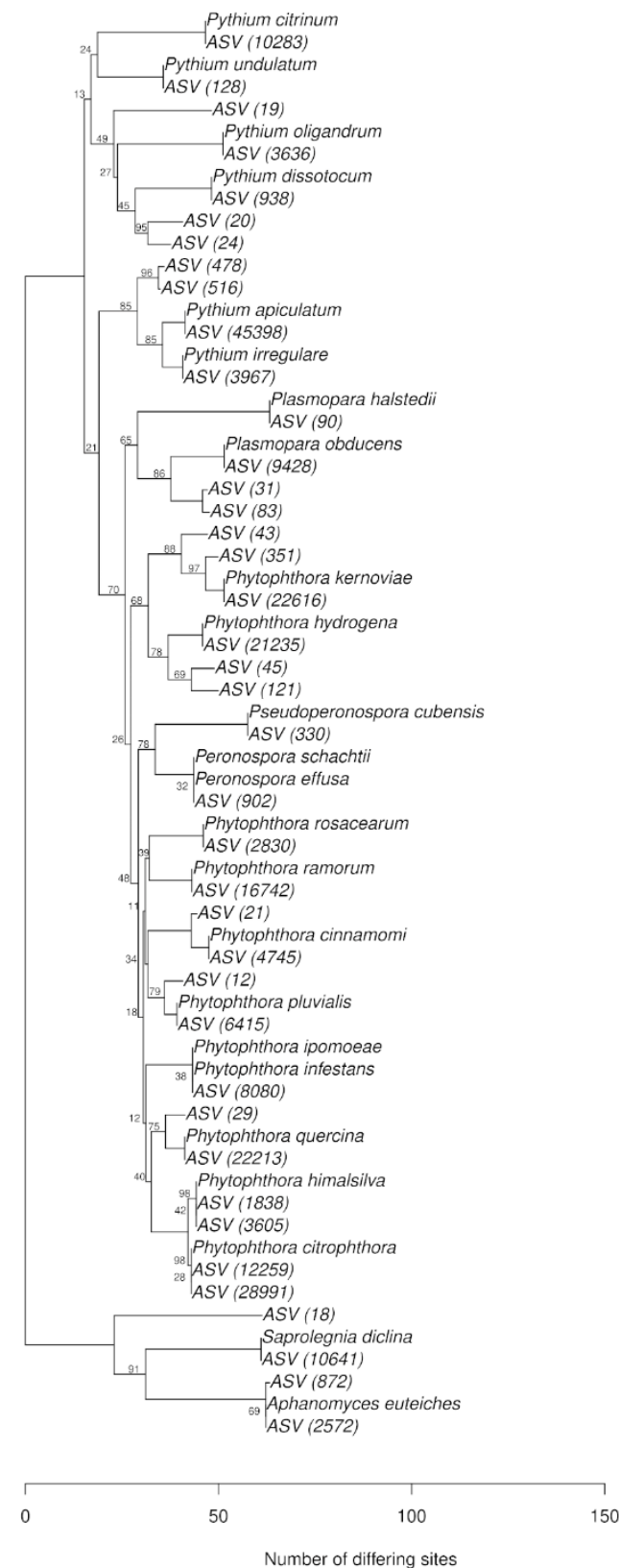

945 Figure S3. Bootstrapped neighbor-joining tree of ASVs in mock community samples and

946 reference sequences for species included in the mock communities. The number of reads

947 represented by each ASV is shown in parentheses. 